\title{
One step cyclocondensation of (thio)barbituric acid with chalcones in glacial acetic acid and phosphorous pentoxide: Part-I
}

\author{
K. Akhter ${ }^{1 *}$, S. M. Ahmed ${ }^{2}$, Md. E. Halim ${ }^{1}$, M. A. Kader ${ }^{1}$, K. Jahan ${ }^{1}$, \\ U. K. R. Romman ${ }^{1}$ and M. G. Ahmed ${ }^{1}$ \\ ${ }^{1}$ Department of Chemistry, University of Dhaka, Dhaka-1000, Bangladesh. \\ ${ }^{2}$ Department of Chemistry, American International University-Bangladesh (AIUB), 83/B, Road-4, Kemal Ataturk \\ Avenue, Banani, Dhaka-1213, Bangladesh
}

\begin{abstract}
Some new 5, 7-diaryl-1,5-dihydro (or 1, 2, 3, 5-tetrahydro)- pyrano[2, 3-d] pyrimidin-2, 4-diones (or 2-thioxo-4-ones) (3a-h) have been synthesized in one-step by cyclocondensation of barbituric acid or thiobarbituric acid (1) with arylideneacetophenones (2a-d), in glacial acetic acid in the presence of phosphorous pentoxide. The structures of the compounds $3 \mathrm{a}-\mathrm{h}$ have been determined by UV, IR, ${ }^{1} \mathrm{H} \mathrm{NMR},{ }^{13} \mathrm{C} \mathrm{NMR}$, mass spectral data and elemental analyses. The compounds $3 \mathrm{a}-\mathrm{h}$ do not seem to be available in the literature.
\end{abstract}

Keywords: Arylideneacetophenone; Barbituric acid; Thiobarbituric acid; Cyclocondensation

\section{Introduction}

The synthesis of fused heterocycles has attracted considerable interest in heterocyclic chemistry as the fusion of biodynamic heterosystems has proved to be very attractive and constructive for the design of a new molecular framework of potential drugs with varying pharmacological activities. Pyran derivatives are ordinary structural subunits in a variety of important natural products, including carbohydrates, alkaloids, polyether antibiotics, pheromones, and iridoids (Tietze et al. 1997). Uracil and its fused derivatives, such as pyrano[2,3-d]pyrimidines, pyrido[2,3-d]pyrimidines or pyrimido[4,5-d]pyrimidines are well recognized by synthesis as well as biological chemists. These annelated uracils have received considerable attention over the past years due to their wide range of biological activity (Senda et al. 1968, Levitt 1982, O'Callaghan et al., 1983, Wrigglesworth et al.1984). Compounds with these ring systems have diverse pharmacological properties such as antiallergic (Kitamura et al., 1984), antihypertensive (Furuya et al. 1994), cardiotonic (Heber et al., 1993), bronchiodilator (Coates 1990), antitumour activity (Broom et al., 1976). Pyrano[2,3-d]pyrimidine is unsaturated six membered heterocycle which is formed by fusion of pyran and pyrimidine rings together, consisting of one oxygen atom at position number 8 and two nitrogen atoms at position number 1 and 3 respectively. If pyrano[2,3- d]pyrimidine moieties are clubbed into one molecule, then resultant derivative enhances its pharmaceutical activity as abundant in biologically active compounds. The synthesis of pyrano[2,3-d]pyrimidines containing a pyran and an uracil ring poses significant synthetic challenges. Therefore, for the preparation of these complex molecules large efforts have been directed towards the synthetic manipulation of uracils. As a result, a number of methods for the synthesis of these compounds have been reported (Rao et al., 1974, Junek et al., 1973, Noboru et al., 1973, Bararjanian et al. 2009, Mohammadi Ziarani et al., 2013), but the majority of them involve various steps, waste of organic solvents, long reaction time and the yields are relatively poor. This initiated to develop an efficient method for the synthesis of these compounds in better yields. Thus new routes for the synthesis of these molecules have attracted considerable attention in search for a rapid entry to these heterocycles. There is a report (Ahlwalia et al., 1993) on the reactions of barbituric acids with $\alpha, \beta$-unsaturated carbonyl systems.

With this background, in continuation to our works (Ahmed et al., 2006, Ahmed et al., 2011, Ullah et al., 2012 and Rahman et al., 2013) on the synthesis of barbituric acid and thio-barbituric acid derivatives, we report herein syntheses

*Corresponding author. e-mail: kawsariakhter@yahoo.com 
of 5, 7-diaryl-1,5-dihydro - pyrano[2, 3- $d$ ] pyrimidin-2, 4diones (3a, 3c, 3e \& 3g) and 5, 7-diaryl-2-thioxo - 1, 2, 3, 5tetrahydro- pyrano[2, 3-d] pyrimidin -4-ones) (3b, 3d, 3f \& $3 \mathrm{~h})$ by selecting a number of arylideneacetophenones (2a- $d$ ) as the $\alpha, \beta$-unsaturated carbonyl system having different substituents on the aromatic rings for reaction with barbituric acid or thiobarbituric acid (1) as the active methylene component in presence of glacial acetic acid and phosphorus pentoxide (Scheme 1). The compounds 3a-h do not seem to be available in the literature.

\section{Materials and methods}

The UV spectra were run in methanol using SHIMADZUUV-160A ultraviolet spectrophotometer with a scanning range of $800-200 \mathrm{~nm}$ using methanol as solvent. The IR spectra were recorded as $\mathrm{KBr}$ pellet using SHIMADZU FTIR 8400 S infra-red spectrophotometer in the range of 4000$400 \mathrm{~cm}^{-1}$. The ${ }^{1} \mathrm{H}$ - and ${ }^{13} \mathrm{C}$ - NMR spectra were recorded on $600 \mathrm{MHz}$ NMR spectrometer. The solvent used was $\mathrm{d}_{6}$ DMSO and TMS is being used as a reference. All the compounds gave expected $\mathrm{C}, \mathrm{H}$ and $\mathrm{N}$ analyses.

3-(4-chlorophenyl)-1-(4-chlorophenyl)-propenone 2a, 3-(4chlorophenyl)-1-(4-aminophenyl)-propenone $2 b, \quad 3-(2-$ chlorophenyl)-1-(4-chlorophenyl)-propenone $2 \mathrm{c}$ and 3-(2chlorophenyl)-1-(4-methoxyphenyl)-propenone $2 \mathrm{~d}$ were prepared from the reactions of corresponding substituted aldehydes and substituted acetophenones by following primarily literature method (Furniss et al. 1978) with modification of the reaction conditions wherever necessary. The reactions described in the present paper were carried out following a general procedure (Ahlwalia et al., 1993).

\section{General procedure}

A mixture of arylideneacetophenone $(0.005 \mathrm{~mol})$ and barbituric acid or thiobarbituric acid $(0.005 \mathrm{~mol})$ were dissolved in acetic acid $(15 \mathrm{~mL})$ and $\mathrm{P}_{2} \mathrm{O}_{5}(1.5 \mathrm{~g})$ in a round-bottomed flask equipped with a magnetic stirrer, a refluxing condenser and a drying tube. The reaction mixture was refluxed at $135-140^{\circ} \mathrm{C}$ for $6-8$ hours and the course of the reaction was followed by TLC on silica gel plates (eluting solvent; $\left.\mathrm{CH}_{3} \mathrm{OH}: \mathrm{CHCl}_{3}=1: 9\right)$. The mixture was allowed to cool and treated with crushed ice. The solid, thus obtained, was filtered off, washed with cold water, dried and purified by recrystallization from rectified spirit.

5,7-bis-(4-chlorophenyl-1,5-dihydro-pyrano[2,3-d]pyrimidine-2,4-dione, $3 a$

White solid; Yield 58\%; mp. $267-269^{\circ} \mathrm{C} ; \mathrm{R}_{\mathrm{f}} 0.78$; UV: $\lambda_{\max }$ $\mathrm{nm} 288,474\left(\pi \rightarrow \pi^{*} / \mathrm{n} \rightarrow \pi^{*}\right.$ of C=O); IR: $\gamma_{\max }\left(\mathrm{cm}^{-1}\right) 3460$ $(\mathrm{N}-\mathrm{H}), 1724,1682(\mathrm{C}=\mathrm{O}$, non-conj.), 1610, $1541 \quad(\mathrm{C}=\mathrm{O}$ arom, C-N) 1437, 1276, 1244, 1176, 1039, 1016, 970, 819 $\left(\mathrm{C}=\mathrm{C}\right.$, arom. \& bar. acid moieties), 1120, 1087 (C-O-C); ${ }^{1} \mathrm{H}$

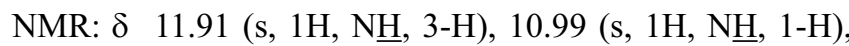
7.69 (d, J $\left.\mathrm{J}_{8.4}, 2 \mathrm{H}, \mathrm{H}-2^{\prime}, 6^{\prime}\right), 7.50$ (d, J $\left.7.8,2 \mathrm{H}, \mathrm{H}-3^{\prime}, 5^{\prime}\right), 7.29-$ 7.34 (m, 4H, H-2",3",5",6"), 6.07 (d, J J.6, 1H, 6-H), 4.34 (d, $\left.\mathrm{J}_{4.5}, 1 \mathrm{H}, 5-\mathrm{H}\right) ;{ }^{13} \mathrm{C}$ NMR: $\delta 163.21$ (4-C), 154.35 (9-C), 144.18 (7-C), 143.22 (2-C), 133.77, 131.10, 130.22, 129.64, 128.68, 128.26, 125.92 (aromatic carbons), 104.64 (6-C), 87.01 (10-C), 34.36 (5-C); MS: m/z $386.99\left(\mathrm{M}^{+}\right), 307.1$, 289.05, 274.99, 154.0 (100\%), 136.0, 89.2; Anal. Found: C, 58.67; H, 3.14; N, 7.04; Calcd. for $\mathrm{C}_{19} \mathrm{H}_{12} \mathrm{~N}_{2} \mathrm{O}_{3} \mathrm{Cl}_{2}: \mathrm{C}$, $58.93 ; \mathrm{H}, 3.12 ; \mathrm{N}, 7.23 \%$.

5,7-bis-(4-chlorophenyl)-2-thioxo-1,2,3,5-tetrahydro-pyrano[2,3-d]pyrimidin-4-one, $3 b$

Gray solid; Yield $33 \%$; mp. $279-281^{\circ} \mathrm{C}$; $\mathrm{R}_{\mathrm{f}} 0.75$; UV: $\lambda_{\max }$ $\mathrm{nm} 295,497\left(\pi \rightarrow \pi^{*} / \mathrm{n} \rightarrow \pi^{*}\right.$ of C=O); IR: $\gamma_{\max }\left(\mathrm{cm}^{-1}\right) 3480$ $(\mathrm{N}-\mathrm{H}), 1676(\mathrm{C}=\mathrm{O}$, non-conj.), $1570(\mathrm{C}=\mathrm{O}$ arom, $\mathrm{C}-\mathrm{N})$ $1410,1273,1238,1220,1055,1012,955,914,829(\mathrm{C}=\mathrm{C}$, arom. \& bar. acid moieties), 1136, 1093 (C-O-C); 1H NMR:

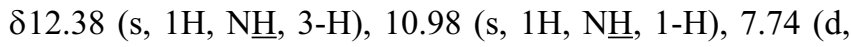
$\left.\mathrm{J}_{8.4}, 2 \mathrm{H}, \mathrm{H}-2^{\prime}, 6^{\prime}\right), 7.50$ (d, $\left.\mathrm{J}_{8.4}, 2 \mathrm{H}, \mathrm{H}-3^{\prime}, 5^{\prime}\right), 7.31-7.35$ (m, 4H, H-2",3",5",6"), 6.09 (d, J $\left.\mathrm{J}_{4.2}, 1 \mathrm{H}, 6-\mathrm{H}\right), 4.49$ (d, J.8, $1 \mathrm{H}, 5-$ H); ${ }^{13}$ C NMR: 160.89 (4-C), 153.68 (9-C), 144.08 (7-C), 173.78 (2-C), 142.58, 133.85, 131.28, 129.97, 129.78, 128.65, 128.28, 125.99 (aromatic carbons), 104.18 (6-C), 92.03 (10-C), 34.29 (5-C); MS: m/z $403.02\left(\mathrm{M}^{+}\right), 371.3$, 307.1, 289.1, 154.0 (100\%), 136.0, 89.3; Anal. Found: C, 55.98; H, 3.78; N, 6.25; Calcd. for $\mathrm{C}_{19} \mathrm{H}_{12} \mathrm{Cl}_{2} \mathrm{~N}_{2} \mathrm{O}_{2} \mathrm{~S}: \mathrm{C}$, $56.59 ; \mathrm{H}, 3.00 ; \mathrm{N}, 6.95 \%$.

7-(4-aminophenyl)-5-(4-chlorophenyl)- 1,5-dihydro-pyrano[2,3-d]pyrimidine-2,4-dione, $3 c$

Red solid; Yield $30 \%$; mp. $264-266^{\circ} \mathrm{C} ; \mathrm{R}_{\mathrm{f}} 0.72$; UV: $\lambda_{\max } \mathrm{nm}$ $320\left(\pi \rightarrow \pi^{*} / \mathrm{n} \rightarrow \pi^{*}\right.$ of $\left.\mathrm{C}=\mathrm{O}\right)$; IR: $\gamma_{\max }\left(\mathrm{cm}^{-1}\right) 3400,3306(\mathrm{~N}-$ $\left.\mathrm{H}, \mathrm{NH}_{2}\right), 1685(\mathrm{C}=\mathrm{O}$, non-conj.), 1595, 1534 ( $\mathrm{C}=\mathrm{O}$ arom, $\mathrm{C}$ - 
N) $1400,1263,1012,831,762(\mathrm{C}=\mathrm{C}$, arom. $\&$ bar. acid moi-

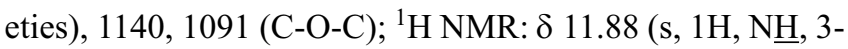
H), 10.98 (s, 1H, N $\underline{H}, 1-\mathrm{H}), 7.72$ (d, 2H, H-2',6'), 7.60 (d, 2H, H-3', 5'), 7.20-7.40 (m, 4H, H-2",3",5",6"), 5.87 (d, $\left.\mathrm{J}_{3.5}, 1 \mathrm{H}, 6-\mathrm{H}\right), 4.44\left(\mathrm{~d}, \mathrm{~J}_{4.0}, 1 \mathrm{H}, 5-\mathrm{H}\right), 3.37$ (s, 2H, Ar- $\left.\underline{\mathrm{H}}_{2}\right)$; ${ }^{13} \mathrm{C}$ NMR: $\delta 161.96$ (4-C), 153.23 (9-C), 145.91 (7-C), 143.77 (2-C), 145.78, 135.80, 130.81, 130.55, 128.78, $127.85,124.90,115.75$ (aromatic carbons), 103.76 (6-C), 87.45 (10-C), 31.85 (5-C); MS: m/z $367.22\left(\mathrm{M}^{+}\right), 307.1$, 289.1, 154.0 (100\%), 136.0, 89.2; Anal. Found: C, 60.30; H, 4.33; N, 7.79; Calcd. for $\mathrm{C}_{19} \mathrm{H}_{14} \mathrm{ClN}_{3} \mathrm{O}_{3}: \mathrm{C}, 62.05 ; \mathrm{H}, 3.84$; $\mathrm{N}, 11.43 \%$.

7-(4-aminophenyl)-5-(4-chlorophenyl)-2-thioxo-1,2,3,5tetrahydro-pyrano[2,3-d] pyrimidine-4-one, $3 d$ :

Red solid; Yield $35 \%$; mp. $248-250^{\circ} \mathrm{C} ; \mathrm{R}_{\mathrm{f}} 0.65$; UV: $\lambda_{\max }$ nm $301\left(\pi \rightarrow \pi^{*} / \mathrm{n} \rightarrow \pi^{*}\right.$ of $\left.\mathrm{C}=\mathrm{O}\right)$; IR: $\gamma_{\max }\left(\mathrm{cm}^{-1}\right) 3395,3300$ $\left(\mathrm{N}-\mathrm{H}, \mathrm{NH}_{2}\right), 1687$ (C=O, non-conj.), 1592, $1524(\mathrm{C}=\mathrm{O}$ arom, C-N) 1407, 1310, 1222, 1182, 1013, $827(\mathrm{C}=\mathrm{C}$, arom. \& bar. acid moieties), 1133, 1091 (C-O-C); ${ }^{1} \mathrm{H}$ NMR: $\delta$

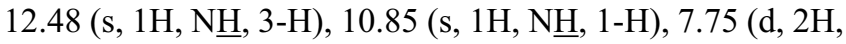
H-2', 6'), 7.68 (d, 2H, H-3', 5'), 7.20-7.40 (m, 4H, H2",3",5",6"), 5.88 (bs, 1H, 6-H), 4.49 (d, J $4.0,1 \mathrm{H}, 5-\mathrm{H}), 3.44$ (s, $\left.2 \mathrm{H}, \mathrm{Ar}-\mathrm{N}_{2}\right) ;{ }^{13} \mathrm{C}$ NMR: $\delta 161.99$ (4-C), 155.47 (9-C), 145.00 (7-C), 173.01 (2-C), 145.90, 135.85, 130.80, 130.60, 128.80, 127.85, 125.00, 113.99 (aromatic carbons), 103.75 (6-C), 88.13 (10-C), 32.28 (5-C); MS: m/z $383.05\left(\mathrm{M}^{+}\right)$, 307.1, 289.1, 154.0 (100\%), 136.0, 89.2; Anal. Found: C, 55.51; H, 3.83; N, 9.08; Calcd. for $\mathrm{C}_{19} \mathrm{H}_{14} \mathrm{ClN}_{3} \mathrm{O}_{2} \mathrm{~S}$ : C, $59.45 ; \mathrm{H}, 3.68 ; \mathrm{N}, 10.95 \%$.

7-(4-chlorophenyl)-5-(2-chlorophenyl)-1,5-dihydro-pyrano[2,3-d]pyrimidine-2,4-dione, 3e:

Light orange solid; Yield 53\%; mp. $306-308^{\circ} \mathrm{C} ; \mathrm{R}_{\mathrm{f}} 0.76$; UV: $\lambda_{\max } \mathrm{nm} 284,348\left(\pi \rightarrow \pi^{*} / \mathrm{n} \rightarrow \pi^{*}\right.$ of $\left.\mathrm{C}=\mathrm{O}\right)$; IR: $\gamma_{\max }\left(\mathrm{cm}^{-1}\right)$ $3393(\mathrm{~N}-\mathrm{H}), 1730(\mathrm{C}=\mathrm{O}$, non-conj.), 1611, $1543(\mathrm{C}=\mathrm{O}$ arom, C-N) 1405, 1255, 1023, 836, $761(\mathrm{C}=\mathrm{C}$, arom. \& bar. acid moieties), 1123, 1077 (C-O-C); ${ }^{1} \mathrm{H}$ NMR: $\delta 11.96$ (s, 1H, $\mathrm{NH}, 3-\mathrm{H}), 11.02$ (s, 1H, Nㅍ, 1-H), 7.68 (d, J $\left.8.4,2 \mathrm{H}, \mathrm{H}-2^{\prime}, 6^{\prime}\right)$, 7.47 (d, J8.4, 2H, H-3', 5'), 7.21-7.42 (m, 4H, H-2",3",5",6") $5.98\left(\mathrm{~d}, \mathrm{~J}_{3.5}, 1 \mathrm{H}, 6-\mathrm{H}\right), 4.85\left(\mathrm{~d}, \mathrm{~J}_{4.2}, 1 \mathrm{H}, 5-\mathrm{H}\right) ;{ }^{13} \mathrm{C}$ NMR: $\delta$ 163.02 (4-C), 155.08 (9-C), 149.63 (7-C), 144.59 (2-C), $140.79,133.83,131.88,130.11,129.71,129.37,128.64$, 128.27, 127.68, 125.97 (aromatic carbons), 102.67 (6-C),
85.94 (10-C), 32.44 (5-C); MS: m/z $387.01\left(\mathrm{M}^{+}\right), 289.1$, 238.1, 154.0 (100\%), 136.0, 89.2, 57.4; Anal. Found: C, 58.33; H, 3.20; N, 7.22; Calcd. for $\mathrm{C}_{19} \mathrm{H}_{12} \mathrm{Cl}_{2} \mathrm{~N}_{2} \mathrm{O}_{3}$ : C, 58.33; $\mathrm{H}, 3.12 ; \mathrm{N}, 7.23 \%$.

7-(4-chlorophenyl)-5-(2-chlorophenyl)-2-thioxo-1,2,3,5tetrahydro-pyrano[2,3-d]pyrimidine-4-one, $3 f$ :

White solid; Yield 57\%; mp. 313-315 ${ }^{\circ}$; $\mathrm{R}_{\mathrm{f}} 0.68$; UV: $\lambda_{\max }$ $\mathrm{nm} 290,359\left(\pi \rightarrow \pi^{*} / \mathrm{n} \rightarrow \pi^{*}\right.$ of C=O); IR: $\gamma_{\max } \quad\left(\mathrm{cm}-{ }^{1}\right) 3369$ $(\mathrm{N}-\mathrm{H}), 1681(\mathrm{C}=\mathrm{O}$, non-conj.), 1615, $1568(\mathrm{C}=\mathrm{O}$ arom, $\mathrm{C}-$ N) $1411,1274,1221,835,757(\mathrm{C}=\mathrm{C}$, arom. \& bar. acid moi-

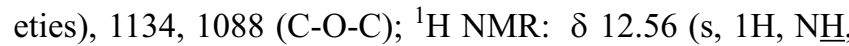

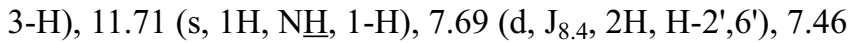
(d, J $\left.{ }_{8.4}, 2 \mathrm{H}, \mathrm{H}-3^{\prime}, 5^{\prime}\right), 7.20-7.43$ (m, 4H, H-2",3",5",6"), 5.95 $\left(\mathrm{d}, \mathrm{J}_{3.5}, 1 \mathrm{H}, 6-\mathrm{H}\right), 4.75\left(\mathrm{~d}, \mathrm{~J}_{4.2}, 1 \mathrm{H}, 5-\mathrm{H}\right) ;{ }^{13} \mathrm{C}$ NMR: $\delta 163.20$ (4-C), 155.80 (9-C), 148.61 (7-C), 174.59 (2-C), 139.80, $133.73,131.78,130.10,129.70,129.27,128.75,128.20$, 127.70, 125.51 (aromatic carbons), 102.62 (6-C), 86.15 (10C), 32.40 (5-C); MS: m/z $403.00\left(\mathrm{M}^{+}\right), 371.3,289.1,154.0$ (100\%), 136.0, 89.2; Anal. Found: C, 56.28; H, 2.90; N, 6.73; Calcd. for $\mathrm{C}_{19} \mathrm{H}_{12} \mathrm{Cl}_{2} \mathrm{~N}_{2} \mathrm{O}_{2} \mathrm{~S}$ : C, 56.59; H, 3.00; N, $6.95 \%$.

5-(2-chlorophenyl)-7-(4-methoxyphenyl)-1,5-dihydro-pyrano[2,3-d]pyrimidine-2,4-dione, $3 g$ :

Brown solid; Yield $60 \%$; mp. $283-285^{\circ} \mathrm{C} ; \mathrm{R}_{\mathrm{f}} 0.70$; UV: $\lambda_{\max }$ $\mathrm{nm} 285,350\left(\pi \rightarrow \pi^{*} / \mathrm{n} \rightarrow \pi^{*}\right.$ of $\left.\mathrm{C}=\mathrm{O}\right)$; IR: $\gamma_{\max }\left(\mathrm{cm}^{-1}\right) 3402$ $(\mathrm{N}-\mathrm{H}), 1725,1683(\mathrm{C}=\mathrm{O}$, non-conj.), $1606(\mathrm{C}=\mathrm{O}$ arom, $\mathrm{C}-$ N) $1441,1307,1252,1035,968,756(\mathrm{C}=\mathrm{C}$, arom. \& bar. acid moieties), 1145, 1070 (C-O-C); 1H NMR: $\delta 11.06$ (s,

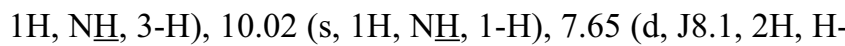
$\left.2^{\prime}, 6^{\prime}\right), 7.50\left(\mathrm{~d}, \mathrm{~J}_{7.9}, 2 \mathrm{H}, \mathrm{H}-3^{\prime}, 5^{\prime}\right), 7.30-7.41(\mathrm{~m}, 4 \mathrm{H}, \mathrm{H}-$ 2",3",5",6"), $5.70\left(\mathrm{~d}, \mathrm{~J}_{3.7}, 1 \mathrm{H}, 6-\mathrm{H}\right), 4.55\left(\mathrm{~d}, \mathrm{~J}_{4.4}, 1 \mathrm{H}, 5-\mathrm{H}\right)$, 3.49 (s, 3H, Ar-OCH ${ }_{3}$ ); ${ }^{13} \mathrm{C}$ NMR: $\delta 166.40$ (4-C), 153.50 (9-C), 149.60 (7-C), 145.59 (2-C), 160.53, 139.80, 134.50 , $130.87,129.70,128.75,127.28,126.95,125.99,113.77$ (aromatic carbons), 107.65 (6-C), 86.65 (10-C), 35.44 (5-C), $55.70\left(\mathrm{Ar}^{-} \mathrm{OCH}_{3}\right) ; \mathrm{MS}: \mathrm{m} / \mathrm{z} 382.04\left(\mathrm{M}^{+}\right), 383.0(100 \%)$, 271.0, 255.0, 135.0, 88.3; Anal. Found: C, 62.14; H, 4.00; N, 7.24; Calcd. for $\mathrm{C}_{20} \mathrm{H}_{15} \mathrm{ClN}_{2} \mathrm{O}_{4}$ : C, 62.75; H, 3.95; N, 7.32\%.

5-(2-chlorophenyl)-7-(4-methoxyphenyl)-2-thioxo-1,2,3,5tetrahydro-pyrano[2,3-d]pyrimidin-4-one, 3h:

Light brown solid; Yield 58\%; mp. $268-270^{\circ} \mathrm{C} ; \mathrm{R}_{\mathrm{f}} 0.71$; UV: 
$\lambda_{\text {max }} \mathrm{nm} 288,358\left(\pi \rightarrow \pi^{*} / \mathrm{n} \rightarrow \pi^{*}\right.$ of $\left.\mathrm{C}=\mathrm{O}\right)$; IR: $\gamma_{\max }\left(\mathrm{cm}^{-1}\right)$ $3384(\mathrm{~N}-\mathrm{H}), 1690(\mathrm{C}=\mathrm{O}$, non-conj.), 1610, $1556(\mathrm{C}=\mathrm{O}$ arom, C-N) 1420, 1257, 1038, 914, 835, $756(\mathrm{C}=\mathrm{C}$, arom. \& bar. acid moieties), 1132, 1071 (C-O-C); ${ }^{1} \mathrm{H}$ NMR: $\delta 12.96$ (s, 1H, NH, 3-H), 11.92 (s, 1H, N $\underline{H}, 1-\mathrm{H}), 7.63$ (d, J ${ }_{8.1}, 2 \mathrm{H}, \mathrm{H}-$ 2',6'), 7.49 (d, J $\left.7.8,2 \mathrm{H}, \mathrm{H}-3^{\prime}, 5^{\prime}\right), 7.22-7.40$ (m, 4H, H2",3",5",6"), 5.69 (d, J3.7,1H, 6-H), 4.65 (d, J4.4,1H, 5-H); ${ }^{13} \mathrm{C}$ NMR: $\delta 165.30$ (4-C), 156.28 (9-C), 149.65 (7-C), 182.59 (2-C), 160.63, 139.82, 134.60, 131.07, 129.65, 128.77, 127.29, 126.93, 126.10, 113.87 (aromatic carbons), 107.63 (6-C), 87.85 (10-C), 34.44 (5-C), $55.70\left(\mathrm{Ar}-\mathrm{OCH}_{3}\right)$; MS: m/z $398.05\left(\mathrm{M}^{+}\right)$, 399.05, $307.1,154$ (100\%), 136.0, 89.2, 57.4; Anal. Found: C, 60.11; H, 3.76; N, 6.98; Calcd. for $\mathrm{C}_{20} \mathrm{H}_{15} \mathrm{ClN}_{2} \mathrm{O}_{3} \mathrm{~S}$ : C, 60.22; H, 3.79; N, 7.02\%.

\section{Results and discussion}

The Compounds 3a-h have been synthesized from 1 and the corresponding $2 \mathrm{a}-\mathrm{d}$ in presence of glacial acetic acid and $\mathrm{P}_{2} \mathrm{O}_{5}$ under refluxing conditions in an analogous manner reported previously (Ahlwalia et al., 1993). The Compounds $3 \mathrm{a}-\mathrm{h}$ have been characterized on the basis of their UV/Vis, IR, ${ }^{1} \mathrm{H}$ NMR, ${ }^{13} \mathrm{C}$ NMR, mass and elemental analyses. The formation of compounds 3a-h may be explained by the initial formation of a 1:1 adduct (A) followed by cyclocondensation (Scheme 1). The formation of such an adduct has been reported (Kharchenko et al., 1976) in the literature.

In their UV spectra of compounds $3 \mathrm{a}-\mathrm{h}$ the observed $\lambda_{\max }$ values agree well to the expected values. The absorption bands in the range $312-286 \mathrm{~nm}$ may be assigned to the $\pi \rightarrow \pi^{*}$ of $\mathrm{C}=\mathrm{O}$ in these compounds. The weak $n \rightarrow \pi^{*}$ absorption bands in the cases of these compounds due to $\mathrm{C}=\mathrm{O}$ were probably masked within the $\pi \rightarrow \pi^{*}$ absorption range of 312 $286 \mathrm{~nm}$.

The IR data of the compounds 3a-h (Table II) showed sharp as well as broad bands in the range $\left(v_{\max }\right) 3480-3300 \mathrm{~cm}^{-1}$ indicating the presence of N-H group. The absorption bands at $1730-1676 \mathrm{~cm}^{-1}$ indicate the presence of non-conjugated $\mathrm{C}=\mathrm{O}$ stretching present in the barbituric acid moieties (Bojarski et al.1985). The bands at $1615-1524 \mathrm{~cm}^{-1}$ were assigned to $\mathrm{C}=\mathrm{C}$ of aromatic rings and $\mathrm{C}=\mathrm{N}$ of the conjugated form of barbituric acid part. Additional bands were observed at $1441-756 \mathrm{~cm}^{-1}$ due to these structural units (Bojarski et al.,1985).

\section{Scheme 1: Synthesis of Pyrmo[2,3-d]pyinidines}

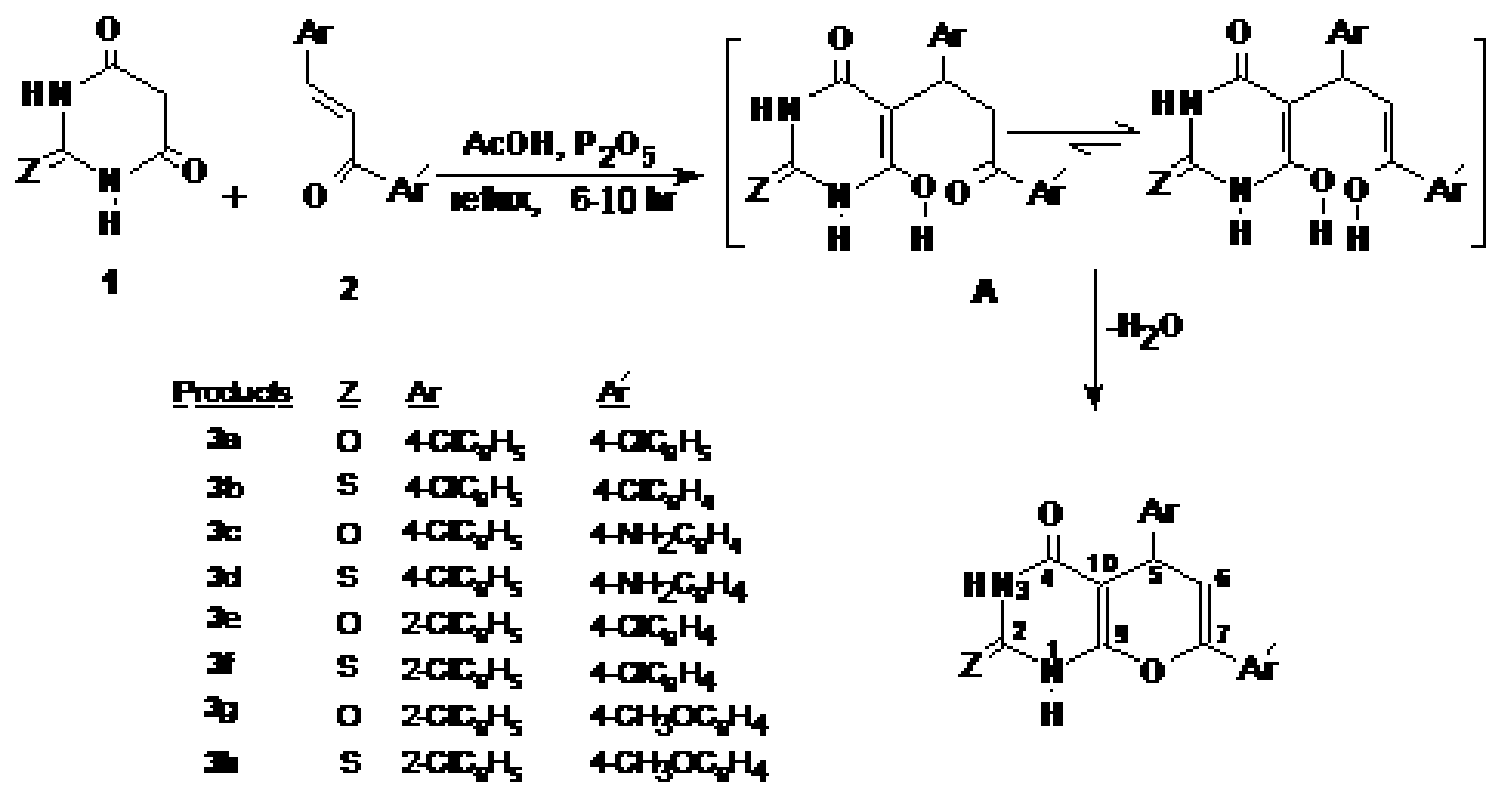

3ah

(1:1matarian) 


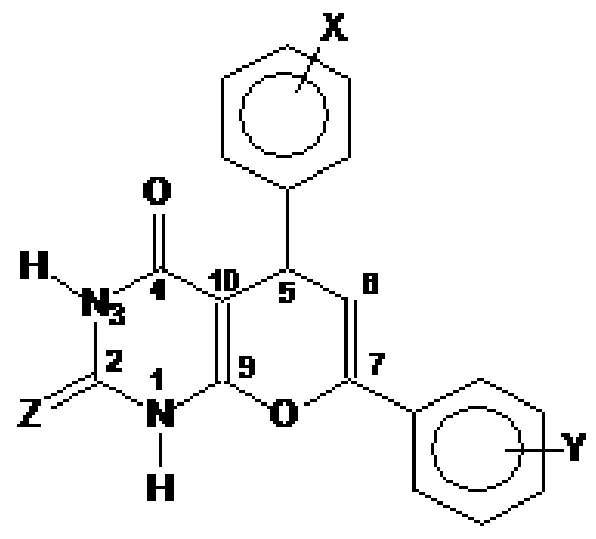

3at

\begin{tabular}{lcccccccc}
\hline Substituent & $3 \mathrm{a}$ & $3 \mathrm{~b}$ & $3 \mathrm{c}$ & $3 \mathrm{~d}$ & $3 \mathrm{e}$ & $3 \mathrm{f}$ & $3 \mathrm{~g}$ & $3 \mathrm{~h}$ \\
\hline $\mathrm{X}$ & $4-\mathrm{Cl}$ & $4-\mathrm{Cl}$ & $4-\mathrm{Cl}$ & $4-\mathrm{Cl}$ & $2-\mathrm{Cl}$ & $2-\mathrm{Cl}$ & $2-\mathrm{Cl}$ & $2-\mathrm{Cl}$ \\
$\mathrm{Y}$ & $4-\mathrm{Cl}$ & $4-\mathrm{Cl}$ & $4-\mathrm{NH}_{2}$ & $4-\mathrm{NH}_{2}$ & $4-\mathrm{Cl}$ & $4-\mathrm{Cl}$ & $4-\mathrm{OCH}_{3}$ & $4-\mathrm{OCH}_{3}$ \\
$\mathrm{Z}$ & $\mathrm{O}$ & $\mathrm{S}$ & $\mathrm{O}$ & $\mathrm{S}$ & $\mathrm{O}$ & $\mathrm{S}$ & $\mathrm{O}$ & $\mathrm{S}$ \\
\hline
\end{tabular}

Table I. Reaction conditions and analytical data of the compounds 3a-h

\begin{tabular}{|c|c|c|c|c|c|c|c|}
\hline Compound & $\begin{array}{l}\text { Reflux } \\
\text { time (hr) }\end{array}$ & $\begin{array}{l}\text { Reaction } \\
\text { temp. }\left({ }^{\circ} \mathrm{C}\right)\end{array}$ & $\begin{array}{l}\text { \% C Found } \\
\text { (Calcd) }\end{array}$ & $\begin{array}{c}\text { \% H Found } \\
\text { (Calcd) }\end{array}$ & $\begin{array}{l}\% \mathrm{~N} \text { Found } \\
\text { (Calcd) }\end{array}$ & Mol. formula & $\begin{array}{l}\mathrm{MS} \\
(\mathrm{m} / \mathrm{z})\end{array}$ \\
\hline $3 a$ & 8 & 135 & $\begin{array}{c}58.67 \\
(58.93)\end{array}$ & $\begin{array}{c}3.14 \\
(3.12)\end{array}$ & $\begin{array}{c}7.04 \\
(7.23)\end{array}$ & $\mathrm{C}_{19} \mathrm{H}_{12} \mathrm{~N}_{2} \mathrm{O}_{3} \mathrm{Cl}_{2}$ & 386.99 \\
\hline $3 b$ & 8 & 140 & $\begin{array}{c}55.98 \\
(56.59)\end{array}$ & $\begin{array}{l}3.78 \\
(3.00)\end{array}$ & $\begin{array}{l}6.25 \\
(6.95)\end{array}$ & $\mathrm{C}_{19} \mathrm{H}_{12} \mathrm{~N}_{2} \mathrm{O}_{2} \mathrm{Cl}_{2} \mathrm{~S}$ & 403.02 \\
\hline $3 c$ & 7 & 137 & $\begin{array}{c}60.30 \\
(62.05)\end{array}$ & $\begin{array}{l}4.33 \\
(3.84)\end{array}$ & $\begin{array}{l}7.79 \\
(11.43)\end{array}$ & $\mathrm{C}_{19} \mathrm{H}_{14} \mathrm{~N}_{3} \mathrm{O}_{3} \mathrm{Cl}$ & 367.22 \\
\hline $3 d$ & 6.5 & 138 & $\begin{array}{l}55.51 \\
(59.45)\end{array}$ & $\begin{array}{c}3.83 \\
(3.68)\end{array}$ & $\begin{array}{l}9.08 \\
(10.95)\end{array}$ & $\mathrm{C}_{19} \mathrm{H}_{14} \mathrm{~N}_{3} \mathrm{O}_{2} \mathrm{ClS}$ & 383.05 \\
\hline $3 e$ & 6 & 140 & $\begin{array}{c}58.33 \\
(58.33)\end{array}$ & $\begin{array}{c}3.20 \\
(3.12)\end{array}$ & $\begin{array}{l}7.22 \\
(7.23)\end{array}$ & $\mathrm{C}_{19} \mathrm{H}_{12} \mathrm{~N}_{2} \mathrm{O}_{3} \mathrm{Cl}_{2}$ & 387.01 \\
\hline $3 \mathrm{f}$ & 6 & 140 & $\begin{array}{c}56.28 \\
(56.59)\end{array}$ & $\begin{array}{l}2.90 \\
(3.00)\end{array}$ & $\begin{array}{l}6.73 \\
(6.95)\end{array}$ & $\mathrm{C}_{19} \mathrm{H}_{12} \mathrm{~N}_{2} \mathrm{O}_{2} \mathrm{Cl}_{2} \mathrm{~S}$ & 403.00 \\
\hline $3 g$ & 7.5 & 135 & $\begin{array}{l}62.14 \\
(62.75)\end{array}$ & $\begin{array}{l}4.00 \\
(3.95)\end{array}$ & $\begin{array}{l}7.24 \\
(7.32)\end{array}$ & $\mathrm{C}_{20} \mathrm{H}_{15} \mathrm{~N}_{2} \mathrm{O}_{4} \mathrm{Cl}$ & 382.04 \\
\hline $3 \mathrm{~h}$ & 7 & 136 & $\begin{array}{c}60.11 \\
(60.22)\end{array}$ & $\begin{array}{c}3.76 \\
(3.79)\end{array}$ & $\begin{array}{l}6.98 \\
(7.02)\end{array}$ & $\mathrm{C}_{20} \mathrm{H}_{15} \mathrm{~N}_{2} \mathrm{O}_{3} \mathrm{Cl} \mathrm{S}$ & 398.05 \\
\hline
\end{tabular}


Table II. Physical constants, IR and UV of compounds 3a-h

\begin{tabular}{|c|c|c|c|c|c|c|c|c|c|}
\hline \multirow{2}{*}{ 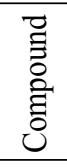 } & \multirow{2}{*}{$\begin{array}{l}\text { m.p. } \\
\left({ }^{\circ} \mathrm{C}\right)\end{array}$} & \multirow{2}{*}{$\begin{array}{c}\text { Yield } \\
(\%)\end{array}$} & \multirow{2}{*}{$\begin{array}{c}\mathrm{R}_{\mathrm{f}} \text { value } \\
\left(\mathrm{CH}_{3} \mathrm{OH}:\right. \\
\mathrm{CHCl}_{3} \\
1: 9) \\
\end{array}$} & \multicolumn{5}{|c|}{$\mathrm{IR}, v_{\max }$ in $\mathrm{cm}^{-1}$} & \multirow{2}{*}{$\begin{array}{c}\mathrm{UV}, \lambda_{\text {max }} \\
(\mathrm{nm}) \\
\pi \rightarrow^{*} \\
\mathrm{n} \rightarrow \pi^{*}\end{array}$} \\
\hline & & & & $\begin{array}{l}\mathrm{N}-\mathrm{H} / \\
\mathrm{NH}_{2}\end{array}$ & $\begin{array}{c}\mathrm{C}=\mathrm{O} \\
\text { non-conj. }\end{array}$ & $\begin{array}{c}\mathrm{C}=\mathrm{O} \\
\text { arom, } \mathrm{C}-\mathrm{N}\end{array}$ & $\begin{array}{c}\mathrm{C}=\mathrm{C} \\
\text { (arom. \& bar. acid moieties) }\end{array}$ & $\mathrm{C}-\mathrm{O}-\mathrm{C}$ & \\
\hline $3 a$ & $267-269$ & 58 & 0.78 & 3460 & $\begin{array}{l}1724 \\
1682\end{array}$ & $\begin{array}{l}1610 \\
1541\end{array}$ & $\begin{array}{c}1437,1276,1244 \\
1176,1039,1016 \\
970,819\end{array}$ & $\begin{array}{r}1120 \\
1087\end{array}$ & 288,474 \\
\hline $3 b$ & $279-281$ & 33 & 0.75 & 3480 & 1676 & 1570 & $\begin{array}{c}1410,1273,1238 \\
1220,1055,1012 \\
955,914,829\end{array}$ & $\begin{array}{l}1136, \\
1093\end{array}$ & 295,497 \\
\hline $3 \mathrm{c}$ & $264-266$ & 30 & 0.72 & $\begin{array}{l}3400 \\
3306\end{array}$ & 1685 & $\begin{array}{l}1595 \\
1534\end{array}$ & $\begin{array}{c}1400,1263,1012 \\
831,762\end{array}$ & $\begin{array}{l}1140 \\
1091\end{array}$ & 320 \\
\hline $3 d$ & $248-250$ & 35 & 0.65 & $\begin{array}{l}3395 \\
3300\end{array}$ & 1687 & $\begin{array}{l}1592 \\
1524\end{array}$ & $\begin{array}{c}1407,1310,1222 \\
1182,1013,827\end{array}$ & $\begin{array}{l}1133, \\
1091\end{array}$ & 301 \\
\hline $3 \mathrm{e}$ & $306-308$ & 53 & 0.76 & 3393 & 1730 & $\begin{array}{l}1611 \\
1543\end{array}$ & $\begin{array}{c}1405,1255,1023, \\
836,761\end{array}$ & $\begin{array}{l}1123 \\
1077\end{array}$ & 284,348 \\
\hline $3 \mathrm{f}$ & $313-315$ & 57 & 0.68 & 3369 & 1681 & $\begin{array}{l}1615 \\
1568\end{array}$ & $\begin{array}{c}1411,1274,1221, \\
835,757\end{array}$ & $\begin{array}{r}1134, \\
1088\end{array}$ & 290,359 \\
\hline $3 g$ & $283-285$ & 60 & 0.70 & 3402 & $\begin{array}{l}1725 \\
1683\end{array}$ & 1606 & $\begin{array}{c}1441,1307,1252 \\
1035,968,756\end{array}$ & $\begin{array}{r}1145 \\
1070\end{array}$ & 285,350 \\
\hline $3 \mathrm{~h}$ & $268-270$ & 58 & 0.71 & 3384 & 1690 & $\begin{array}{l}1610 \\
1556\end{array}$ & $\begin{array}{c}1420,1257,1038 \\
914,835,756\end{array}$ & $\begin{array}{l}1132 \\
1071\end{array}$ & 288,358 \\
\hline
\end{tabular}

The N-H protons at positions 1 and 3 in the compounds $3 \mathrm{a}-$ $\mathrm{h}$ were strongly deshielded ( $\delta$ 12.56-10.02) and appeared as singlet in their ${ }^{1} \mathrm{H}$ NMR spectra (Table III). The N-H protons at position 3 in these compounds were found comparatively more deshielded than protons at position 1. In some compounds ( $3 \mathrm{~b}, 3 \mathrm{~d}, 3 \mathrm{f} \& 3 \mathrm{~h}$ ) more deshielding of the N-H protons were observed due to presence of thiocarbonyl group. This may be attributed to the greater polarizability of sulfur in comparison to oxygen.

The proton at position 6 in 3a-h appeared as a doublet (or broad singlet) due to the vicinal coupling with the proton at position 5. The chemical shifts were observed at $\delta$ 6.09-5.69. The 6-H in these compounds gave signals at $\delta 4.85-4.44$ as doublet due to the coupling received from the proton at position 5 .
The chemical shifts for the aromatic protons in 3a-h were found in good agreement with the literature values (Silverstein et al., 1991 and Kemp 1991).

The structures of the compounds 3a-h were further confirmed by their ${ }^{13} \mathrm{C}$ NMR spectra (Table IV). The chemical shifts of carbonyl carbon at 4-C were found to be deshielded in the range of $\delta 166.40-160.89$. The chemical shifts of 9-C were also deshielded ( $\delta 156.28-153.23$ ). This value is comparable with the ${ }^{13} \mathrm{C}$ NMR chemical shifts of cyclohexyl methyl ketone (Marr et al., 1965).

In the compounds $3 \mathrm{a}, 3 \mathrm{c}, 3 \mathrm{e}$ and $3 \mathrm{~g}$, the chemical shifts of carbonyl carbons at 2-C were found to be at $\delta$ 145.59-143.22 and are relatively less deshielded due to the resonance of amide functional group. In the compounds $3 \mathrm{~b}, 3 \mathrm{~d}, 3 \mathrm{f}$ and $3 \mathrm{~h}$, 
Table III. ${ }^{1} \mathrm{H}$ NMR spectral data of the compounds $3 \mathrm{a}-\mathrm{h} .[(\boldsymbol{\delta})$ in ppm]

\begin{tabular}{|c|c|c|c|c|c|c|c|}
\hline Compound & $3-\mathrm{H}$ & $1-\mathrm{H}$ & Aromatic & $6-\mathrm{H}$ & $5-\mathrm{H}$ & $\mathrm{X}$ & $\mathrm{Y}$ \\
\hline $3 a$ & $\begin{array}{c}11.91 \\
(\mathrm{~s}, 1 \mathrm{H}, \mathrm{N} \underline{\mathrm{H}})\end{array}$ & $\begin{array}{c}10.99 \\
(\mathrm{~s}, 1 \mathrm{H}, \mathrm{N} \underline{\mathrm{H}})\end{array}$ & $\begin{array}{l}7.69\left(\mathrm{~d}, \mathrm{~J}_{8.4}, 2 \mathrm{H}, \mathrm{H}-2^{\prime}, 6^{\prime}\right) \\
7.50\left(\mathrm{~d}, \mathrm{~J}_{7.8}, 2 \mathrm{H}, \mathrm{H}-3^{\prime}, 5^{\prime}\right) \\
7.29-7.34\left(\mathrm{~m}, 4 \mathrm{H}, \mathrm{H}-2^{\prime \prime}, 3^{\prime \prime}, 5^{\prime \prime}, 6^{\prime \prime}\right)\end{array}$ & $\begin{array}{l}6.07 \\
\left(\mathrm{~d}, \mathrm{~J}_{3.6}, 1 \mathrm{H}\right)\end{array}$ & $\begin{array}{l}4.47 \\
\left(\mathrm{~d}, \mathrm{~J}_{3.6}, 1 \mathrm{H}\right)\end{array}$ & ....... & ...... \\
\hline $3 b$ & $\begin{array}{c}12.38 \\
(\mathrm{~s}, 1 \mathrm{H}, \mathrm{N} \underline{\mathrm{H}})\end{array}$ & $\begin{array}{c}10.98 \\
(\mathrm{~s}, 1 \mathrm{H}, \mathrm{N} \underline{\mathrm{H}})\end{array}$ & $\begin{array}{l}7.74\left(\mathrm{~d}, \mathrm{~J}_{8.4}, 2 \mathrm{H}, \mathrm{H}-2^{\prime}, 6^{\prime}\right) \\
7.50\left(\mathrm{~d}, \mathrm{~J}_{8.4}, 2 \mathrm{H}, \mathrm{H}-3^{\prime}, 5^{\prime}\right) \\
7.31-7.35\left(\mathrm{~m}, 4 \mathrm{H}, \mathrm{H}-2^{\prime \prime}, 3^{\prime \prime}, 5^{\prime \prime}, 6^{\prime \prime}\right)\end{array}$ & $\begin{array}{l}6.09 \\
\left(\mathrm{~d}, \mathrm{~J}_{4.2}, 1 \mathrm{H}\right)\end{array}$ & $\begin{array}{l}4.49 \\
\left(\mathrm{~d}, \mathrm{~J}_{4.2}, 1 \mathrm{H}\right)\end{array}$ & ....... & $\cdots \cdots$ \\
\hline $3 c$ & $\begin{array}{c}11.88 \\
(\mathrm{~s}, 1 \mathrm{H}, \mathrm{N} \underline{\mathrm{H}})\end{array}$ & $\begin{array}{c}10.98 \\
(\mathrm{~s}, 1 \mathrm{H}, \mathrm{N} \underline{\mathrm{H}})\end{array}$ & $\begin{array}{l}7.72\left(\mathrm{~d}, \mathrm{~J}_{8}, 2 \mathrm{H}, \mathrm{H}-3^{\prime \prime}, 5^{\prime \prime}\right) \\
7.60\left(\mathrm{~d}, \mathrm{~J}_{7.2}, 2 \mathrm{H}, \mathrm{H}-2^{\prime}, 6^{\prime}\right) \\
7.30-7.35\left(\mathrm{~m}, 4 \mathrm{H}, \mathrm{H}-2^{\prime \prime}, 3^{\prime}, 5^{\prime}, 6^{\prime \prime}\right)\end{array}$ & $\begin{array}{l}5.87 \\
\left(\mathrm{~d}, \mathrm{~J}_{4.0}, 1 \mathrm{H}\right)\end{array}$ & $\begin{array}{l}4.44 \\
\left(\mathrm{~d}, \mathrm{~J}_{4.0}, 1 \mathrm{H}\right)\end{array}$ & $\ldots \ldots$ & $\begin{array}{c}3.37 \\
\left(\mathrm{Ar}-\mathrm{NH}_{2}\right)\end{array}$ \\
\hline $3 d$ & $\begin{array}{c}12.48 \\
(\mathrm{~s}, 1 \mathrm{H}, \mathrm{N} \underline{\mathrm{H}})\end{array}$ & $\begin{array}{c}10.85 \\
(\mathrm{~s}, 1 \mathrm{H}, \mathrm{NH})\end{array}$ & $\begin{array}{l}7.70\left(\mathrm{~d}, \mathrm{~J}_{8.2}, 2 \mathrm{H}, \mathrm{H}-3^{\prime \prime}, 5^{\prime \prime}\right) \\
7.60\left(\mathrm{~d}, \mathrm{~J}_{7.4}, 2 \mathrm{H}, \mathrm{H}-2^{\prime}, 6^{\prime}\right) \\
7.30-7.35\left(\mathrm{~m}, 4 \mathrm{H}, \mathrm{H}-2^{\prime \prime}, 3^{\prime}, 5^{\prime}, 6^{\prime \prime}\right)\end{array}$ & $\begin{array}{l}5.88 \\
(\mathrm{bs}, 1 \mathrm{H})\end{array}$ & $\begin{array}{l}4.49 \\
\left(\mathrm{~d}, \mathrm{~J}_{4.0}, 1 \mathrm{H}\right)\end{array}$ & $\ldots \ldots$. & $\begin{array}{c}3.44 \\
\left(\mathrm{Ar}-\mathrm{NH}_{2}\right)\end{array}$ \\
\hline $3 e$ & $\begin{array}{c}11.96 \\
(\mathrm{~s}, 1 \mathrm{H}, \mathrm{N} \underline{\mathrm{H}})\end{array}$ & $\begin{array}{c}11.02 \\
(\mathrm{~s}, 1 \mathrm{H}, \mathrm{N} \underline{\mathrm{H}})\end{array}$ & $\begin{array}{l}7.68\left(\mathrm{~d}, \mathrm{~J}_{8.4}, 2 \mathrm{H}, \mathrm{H}-2^{\prime}, 6^{\prime}\right) \\
7.47\left(\mathrm{~d}, \mathrm{~J}_{8.4}, 2 \mathrm{H}, \mathrm{H}-3^{\prime}, 5^{\prime}\right) \\
7.21-7.42\left(\mathrm{~m}, 4 \mathrm{H}, \mathrm{H}-2^{\prime \prime}, 3^{\prime \prime}, 5^{\prime \prime}, 6^{\prime \prime}\right)\end{array}$ & $\begin{array}{l}5.98 \\
\left(\mathrm{~d}, \mathrm{~J}_{4.2}, 1 \mathrm{H}\right)\end{array}$ & $\begin{array}{l}4.85 \\
\left(\mathrm{~d}, \mathrm{~J}_{4.2}, 1 \mathrm{H}\right)\end{array}$ & $\ldots \ldots$ & ....... \\
\hline $3 \mathrm{f}$ & $\begin{array}{c}12.56 \\
(\mathrm{~s}, 1 \mathrm{H}, \mathrm{N} \underline{\mathrm{H}})\end{array}$ & $\begin{array}{c}11.71 \\
(\mathrm{~s}, 1 \mathrm{H}, \mathrm{N} \underline{\mathrm{H}})\end{array}$ & $\begin{array}{l}7.69\left(\mathrm{~d}, \mathrm{~J}_{8.4}, 2 \mathrm{H}, \mathrm{H}-2^{\prime}, 6^{\prime}\right) \\
7.46\left(\mathrm{~d}, \mathrm{~J}_{8.4}, 2 \mathrm{H}, \mathrm{H}-3^{\prime}, 5^{\prime}\right) \\
7.20-7.43\left(\mathrm{~m}, 4 \mathrm{H}, \mathrm{H}-2^{\prime \prime}, 3^{\prime \prime}, 5^{\prime \prime}, 6^{\prime \prime}\right)\end{array}$ & $\begin{array}{l}5.95 \\
\left(\mathrm{~d}, \mathrm{~J}_{4.1}, 1 \mathrm{H}\right)\end{array}$ & $\begin{array}{l}4.75 \\
\left(\mathrm{~d}, \mathrm{~J}_{4.1}, 1 \mathrm{H}\right)\end{array}$ & $\ldots \ldots$ & ....... \\
\hline $3 g$ & $\begin{array}{c}11.06 \\
(\mathrm{~s}, 1 \mathrm{H}, \mathrm{N} \underline{\mathrm{H}})\end{array}$ & $\begin{array}{c}10.02 \\
(\mathrm{~s}, 1 \mathrm{H}, \mathrm{N} \underline{\mathrm{H}})\end{array}$ & $\begin{array}{l}7.65\left(\mathrm{~d}, \mathrm{~J}_{8.1}, 2 \mathrm{H}, \mathrm{H}-2^{\prime}, 6^{\prime}\right) \\
7.50\left(\mathrm{~d}, \mathrm{~J}_{7.9}, 2 \mathrm{H}, \mathrm{H}-3^{\prime}, 5^{\prime}\right) \\
7.30-7.41\left(\mathrm{~m}, 4 \mathrm{H}, \mathrm{H}-2^{\prime \prime}, 3^{\prime \prime}, 5^{\prime \prime}, 6^{\prime \prime}\right)\end{array}$ & $\begin{array}{l}5.70 \\
\left(\mathrm{~d}, \mathrm{~J}_{4.0}, 1 \mathrm{H}\right) \\
\end{array}$ & $\begin{array}{l}4.55 \\
\left(\mathrm{~d}, \mathrm{~J}_{4.0}, 1 \mathrm{H}\right)\end{array}$ & $\ldots \ldots$ & $\begin{array}{c}3.49 \\
\left(\mathrm{Ar}-\mathrm{OCH}_{3}\right)\end{array}$ \\
\hline $3 \mathrm{~h}$ & $\begin{array}{c}12.96 \\
(\mathrm{~s}, 1 \mathrm{H}, \mathrm{N} \underline{\mathrm{H}})\end{array}$ & $\begin{array}{c}11.92 \\
(\mathrm{~s}, 1 \mathrm{H}, \mathrm{N} \underline{\mathrm{H}})\end{array}$ & $\begin{array}{l}7.63\left(\mathrm{~d}, \mathrm{~J}_{8.1}, 2 \mathrm{H}, \mathrm{H}-2^{\prime}, 6^{\prime}\right) \\
7.49\left(\mathrm{~d}, \mathrm{~J}_{7.8}, 2 \mathrm{H}, \mathrm{H}-3^{\prime}, 5^{\prime}\right) \\
7.22-7.40\left(\mathrm{~m}, 4 \mathrm{H}, \mathrm{H}-2^{\prime \prime}, 3^{\prime \prime}, 5^{\prime \prime}, 6^{\prime \prime}\right)\end{array}$ & $\begin{array}{l}5.69 \\
\left(\mathrm{~d}, \mathrm{~J}_{4.1}, 1 \mathrm{H}\right)\end{array}$ & $\begin{array}{l}4.65 \\
\left(\mathrm{~d}, \mathrm{~J}_{4.1}, 1 \mathrm{H}\right)\end{array}$ & $\ldots \ldots$ & $\begin{array}{c}3.50 \\
\left(\mathrm{Ar}-\mathrm{OCH}_{3}\right)\end{array}$ \\
\hline
\end{tabular}
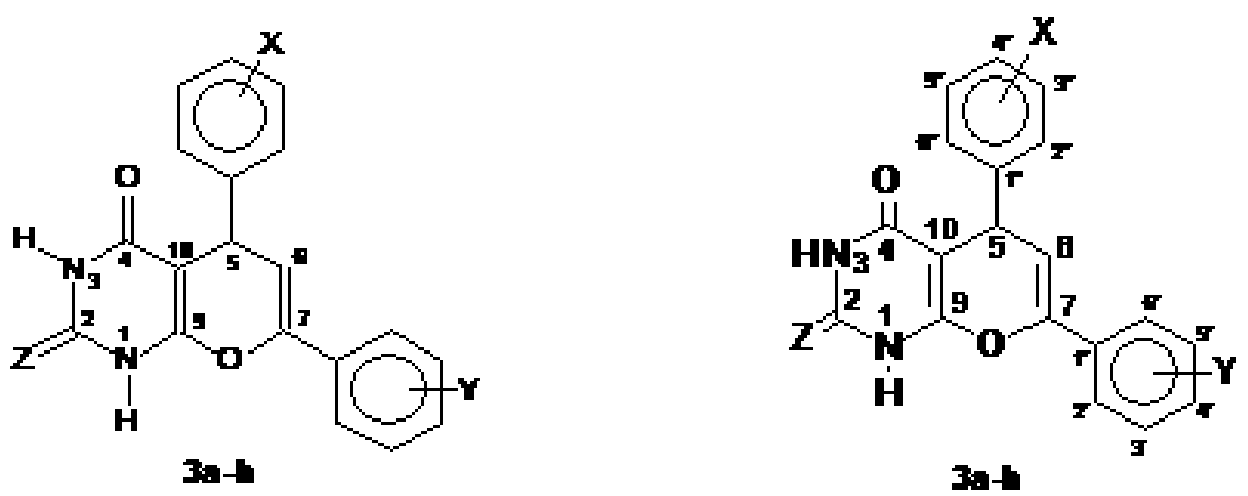
Table IV. ${ }^{13} \mathrm{C}$ NMR spectral data of the compounds 3a-h. [( $\left.\delta\right)$ in ppm]

\begin{tabular}{|c|c|c|c|c|c|c|c|c|c|c|}
\hline Compound & $3-\mathrm{C}$ & $9-\mathrm{C}$ & $7-\mathrm{C}$ & $2-\mathrm{C}$ & Aromatic carbons & $6-\mathrm{C}$ & $10-\mathrm{C}$ & $5-\mathrm{C}$ & $\mathrm{X}$ & Y \\
\hline $3 a$ & 163.21 & 154.35 & 144.18 & 143.22 & $133.77-125.92$ & 104.64 & 87.01 & 34.36 & $\ldots$ & $\ldots$ \\
\hline $3 b$ & 160.89 & 153.68 & 144.08 & 173.78 & $142.58-125.99$ & 104.18 & 92.03 & 34.29 & $\ldots$ & $\ldots$ \\
\hline $3 \mathrm{c}$ & 161.96 & 153.23 & 145.91 & 143.77 & $145.78-115.75$ & 103.76 & 87.45 & 31.85 & $\ldots$ & $\ldots$ \\
\hline $3 \mathrm{~d}$ & 161.99 & 155.47 & 145.00 & 173.01 & $145.90-113.99$ & 103.75 & 88.13 & 32.28 & $\ldots$ & $\ldots$ \\
\hline $3 e$ & 163.02 & 155.08 & 149.63 & 144.59 & $140.79-125.97$ & 102.67 & 85.94 & 32.44 & $\ldots$ & $\ldots$ \\
\hline $3 f$ & 163.20 & 155.80 & 148.61 & 174.59 & $139.80-125.51$ & 102.62 & 86.15 & 32.40 & $\ldots$ & $\ldots$ \\
\hline $3 g$ & 166.40 & 153.50 & 149.60 & 145.59 & $160.53-113.77$ & 107.65 & 86.65 & 35.44 & $\ldots$ & $\begin{array}{c}55.70 \\
\left(\mathrm{Ar}-\mathrm{OCH}_{3}\right)\end{array}$ \\
\hline $3 \mathrm{~h}$ & 165.30 & 156.28 & 149.65 & 182.59 & $160.63-113.87$ & 107.63 & 87.85 & 34.44 & $\ldots$ & $\begin{array}{c}55.70 \\
\left(\mathrm{Ar}-\mathrm{OCH}_{3}\right)\end{array}$ \\
\hline
\end{tabular}

the chemical shifts of thioxo carbon at 2-C were found to be at $\delta$ 182.59-173.01. This explains that the replacement of a carbonyl group by a thiocarbonyl group results in a downfield shift (Otto et al., 1976, Ahmed et al., 2005).

The chemical shift values for 7-C and 6-C in these compounds were observed at $\delta 149.65-144.08$ and $\delta 107.65-$ 103.75 respectively. The $10-\mathrm{C}$ of the compounds showed chemical shift values at $\delta$ 92.03-86.15 which were comparable to the earlier report (Bojarski et al.1985) of the ${ }^{13} \mathrm{C} N M R$ spectral data of the monosubstituted barbiturates at 10-C. The chemical shift values for 5-C in these compounds were observed at $\delta$ 35.44-31.85.

The ${ }^{13} \mathrm{C}$ NMR chemical shifts for the carbons of aromatic rings were assigned on the basis of a correlation chart available in the literature (Levy et al., 1972).

The compounds 3a-h showed peaks for their respective molecular ions $\left(\mathrm{M}^{+}\right)$in their high resolution mass spectra at m/z 386.99 (67\%), 403.02 (20.7\%), 367.22 (5\%), 383.05 (10.0\%), 387.01 (47.4\%), 403.00 (13.7\%), 382.04 (20.0\%) and $398.05(7.0 \%)$ respectively. The isotopic pattern for $\mathrm{Cl}$ atom $\left({ }^{35} \mathrm{Cl} /{ }^{37} \mathrm{Cl}, 3: 1\right)$ was observed in the molecular mass of the compounds $3 \mathrm{a}-\mathrm{h}$.

\section{Acknowledgement}

Authors gratefully acknowledge the help of Prof. Teruo Shinmyozu, Department of Molecular Chemistry, Kyushu
University, Fukuoka, Japan for recording mass spectra and determining elemental analyses of our compounds. Our special thanks goes to S. M. A. Hakim Siddiki, Graduate School of Material Science, University of Hyogo, Japan for recording proton and carbon-13 nmr spectra of the compounds.

\section{References}

Ahmed MG, Romman UKR, Ahmed SM, Akhter K, Halim ME and Salauddin M (2006), A study on the synthesis of 5,7-diaryl-1,2,3,4-tetrahydro-2,4-dioxo-5H-pyrano[2,3-d]pyrimidines; Bangladesh J. Sci. Ind. Res. 41(3-4): 119-128 and references herein.

Ahmed MG, Romman UKR, Akhter K, Halim ME, Rahman MM and Ahmed SM (2011), A one-step synthesis of 5,7-Diaryl-1,5-dihydro (or 1,2,3,5-tetrahydro)-pyrano[2,3- $d]$ pyrimidin-2,4-diones (or 2-thioxo-4-ones), Ind. J. Chem. 50B: 946-948.

Ahmed MG, Ahmed SA, Ahmed SM, Hussam A and Hossain MM (2005), Synthesis of 7,11-diaryl-2,4diazaspiro[5,5] undecane-3-oxo(or thioxo)-1,5,9trines, Part-1. J. Chem. Res., 10: 622-625.

Ahlwalia VK, Aggarwal R and Kumar R (1993), A Convenient One-Pot Synthesis of 5-Aryl-7-methyl1,2,3,4-tetrahydro-2,4-dioxo-5H-pyrano(2,3-d) pyrimidines, Ind. J. Chem., 32B: 963-964.

Bararjanian M, Balalaie S, Movassagh B and Amani AM 
(2009), One-Pot Synthesis of Pyrano[2,3-d]pyrimidinone Derivatives Catalyzed by L-Proline in Aqueous Media, J. Iran. Chem. Soc., 6(2): 436-442.

Broom AD, Shim JL and Anderson GL (1976), Pyrido [2,3d]pyrimidines. IV. Synthetic studies leading to various oxopyrido [2,3-d] pyrimidines. J Org Chem, 41:10951099.

Bojarski JT, Mokrosz JL, Barton HJ and Paluchowaka MH (1985), Advances in Heterocyclic Chemistry (Review Article), 38: 229-297.

Coates WJ (1990), Pyrimidopyrimidine Derivatives. Eur Pat 351058. Chem Abstr, 113: 40711.

Furniss BS, Hannaford AJ, Rogers V, Smith PWG and Tatchell AR (1978), Vogel's Text Book of Practical Organic Chemistry, $4^{\text {th }}$ Ed. (Longman Group Ltd., London) p. 796.

Furuya S and Ohtaki T (1994), Pyridopyrimidine derivatives, their production and use. Eur Pat Appl EP 608565. Chem Abstr, 121:205395w.

Heber D, Heers C and Ravens U (1993), Positive inotropic activity of 5-amino-6-cyano-1, 3-dimethyl-1, 2, 3, 4tetrahydropyrido $[2,3-d]$ pyrimidine-2, 4-dione in cardiac muscle from guinea-pig and man. Part 6: compounds with positive inotropic activity. Die Pharmazie, 48:537-541.

Junek H and Aigner H (1973) Synthesen mit Nitrilen, XXXV. Reaktionen von Tetracyanäthylen mit Heterocyclen. Chem Ber, 106: 914-921.

Kharchenko VG, Markova LI and Korshunova KM (1976), On Reactions ofoxo-1, 5-diketones with sulfurous reagents, Zh. Org. Khim., 12(3): 663; Chem Abstr (1976) 85: 32775c.

Kemp W (1991), Organic Spectroscopy, $3^{\text {rd }}$ Ed., Macmillan, London.

Kitamura N and Onishi A (1984), Eur. Pat. 163599. Chem Abstr, 104:186439.
Levitt G (1982), Herbicidal sulfonamides, US Patent 4339267; Chem Abstr, (1983) 98: 215602g.

Levy GC and Nelson GL (1972), Carbon-13 Nuclear Magnetic Resonance for Organic Chemists, John Wiley \& Sons, N. Y..

Marr DH and Stothers JB (1965), ${ }^{13}$ C NMR Studies: Part VI. Carbon-13 Spectra of $\alpha, \beta$ - Unsaturated Carbonyl Compounds, Canad. J. Chem., 43: 596-607.

Noboru S, Yoshikazu K and Psurematsu T (1973), Chem Pharm Bull, 21: 2639.

Otto HH and Triepel J (1976), Synthesis and structure of 7,11-diphenyl-2,4-diazaspiro[5.5] undecan-1,3,5,9tetraones, I. Liebigs Ann. Chem., 1982-1991.

O'Callaghan CN and Conalty ML (1983), Anticancer Agents: XVII. Synthesis and antitumour activity of 2uyl-4-oxo-2,3-dihydrobenzopyrano[2,3-d] pyrimidines, and 4-substituted 2-aryl-5h-benzopyrano-[2,3d] pyrimidines, Proc. R. Ir. Acad., 83B: 241.

Rahman MM, Ahmed SM, Siddiki SMAH, Halim ME, Akhter K, Romman UKR and Ahmed MG (2013); A one pot synthesis of 5, 7-diaryl-1,5-dihydro (or 1,2,3, 5-tetrahydro)- pyrano[2, 3- $d$ ] pyrimidin-2, 4-diones (or 2-thioxo-4-ones), Dhaka Univ. J. Sc. 61(2): 167171.

Rao AS and Mitra RB (1974), Synthesis of heterocycles. II. Pyrano[2,3-dlpyrimidines. Ind. J. Chem., 12: 1028; Chem Abstr, (1975) 82: 112023.

Senda S, Fujimura H and Izumi H (1968), Barbituric acid analgesics, Japan Patent, 193, 6824; Chem Abstr, (1969) 70: 78001r.

Silverstein RM, Bassler GC and Morill TC (1991), Spectroscopic Identification of Organic Compounds, $5^{\text {th }}$ Ed., John Wiley \& Sons, N.Y..

Tietze LF and Kettschau G (1997), Topics in Current Chemistry, Springer (Berlin) 189:1-120. 
Ullah MS, Ahmed MG, Romman UKR, Akhter K, Ahmed SM and Halim ME (2012), Synthesis of 5, 7-diaryl1,5-dihydro (or 1, 2, 3, 5-tetrahydro)- pyrano[2, 3-d] pyrimidin-2, 4-diones (or 2-thioxo-4-ones), $J$. Bangladesh Chem. Soc. 25(2): 124-131.

Wrigglesworth R, Inglish WD, Livingstone DB, Suekling CJ and Wood HCS (1984), Specific enzyme inhibitors in vitamin biosynthesis. Part 6. Identification of an affinity chromatography ligand for the purification of riboflavin synthase, J. Chem. Soc. (Perkin Trans I), 5: 959-963.
Ziarani GM, Faramarzi S, Asadi S, Badiei A, Bazl R and Amanlou M (2013), Three-component synthesis of pyrano $[2,3-d]$-pyrimidine dione derivatives facilitated by sulfonic acid nanoporous silica (SBA-Pr-SO3H) and their docking and urease inhibitory activity, DARU Journal of Pharmaceutical Sciences, 21:3.

Received: 19 September 2015; Revised: 09 December 2015; Accepted: 31 january 2016. 